\title{
Autonomous High Precision Positioning of Surgical Instruments in Robot-Assisted Minimally Invasive Surgery under Visual Guidance
}

\author{
Christoph Staub, Alois Knoll \\ Robotics and Embedded Systems \\ Technische Universität München \\ Munich, Germany \\ \{staublknoll\}@in.tum.de
}

\author{
Takayuki Osa \\ Department of Engineering Synthesis \\ University of Tokyo \\ Tokyo, Japan \\ osa@nml.t.u-tokyo.ac.jp
}

\author{
Robert Bauernschmitt \\ Department of Cardiovascular Surgery \\ German Heart Center Munich \\ Munich, Germany \\ bauernschmitt@dhm.mhn.de
}

\begin{abstract}
Despite the fact that minimally invasive robotic surgery provides many advantages for patients, complex tasks are still time-consuming, error-prone and lead to quicker fatigue of the surgeon. Automating recurrent tasks could greatly reduce total surgery time for patients. While surgeons gain most of the information which is necessary to perform the operation from the visual feedback of cameras, there is only little work on autonomous systems utilizing visual information to generate movement commands. A major step towards automated tasks is the autonomous positioning of surgical instruments with high precision inside the situs. In this paper, we tackle the challenges arising from automated positioning by employing visual servoing techniques in two ways: On one hand, a calibration of all system components has to be performed to enable position-based servoing in Cartesian space. On the other hand, lever effects which appear due to the nature of laparoscopic surgery and intrinsic system imprecisions may not be overcome with calibrations. Therefore, the instruments can also be servoed image-based. Combining both approaches to a switching scheme allows for autonomous high precision positing of surgical instruments in a complex setup with four robots.
\end{abstract}

Keywords-autonomous positioning; visual servoing; robotic surgery;

\section{INTRODUCTION}

Endoscopic surgery is a challenging technique and has had significant impact on both patients and surgeons. Minimally invasive surgery (MIS) techniques avoid large cuts and patients profit from less pain and collateral trauma. Therefore, the time of hospitalization and the infection rate can be reduced. On the other hand, surgeons have to cope with increasingly complex working conditions. Since endoscopic surgery is performed through small incisions or ports in the body, surgeons must learn to operate with a flattened view caused by the endoscopic image and with unfamiliar surgical instruments. The introduction of telemanipulators, such as the daVinci ${ }^{\mathrm{TM}}$ machine, has overcome these limitations and is a remarkable example of the ongoing research. The instruments can be controlled remotely by a surgeon sitting at a master console, which can be placed somewhere in the operation theater. A stereoscopic endoscope provides a $3 \mathrm{D}$ view on the situs and improves the perceptual limitations of flattened images. The master console is equipped with sophisticated input devices and provides an intuitive handling of the surgical instruments (Cartesian control without any chopstick effect). The robots at the slave system offer as much freedom of movement as the surgeon's own hand would in conventional open surgery. However, these advantages come at the price of reduced immersiveness, since the surgeon cannot feel any forces exerted onto the situs. In addition, working speed is significantly reduced during delicate maneuvers such as knot-tying [1].

In order to research on the improvement of these disadvantages, we have developed an experimental system for MIS incorporating force feedback [2] which is capable of learning a knot, demonstrated by an expert (the surgeon), utilizing a scaffolding framework [3], [4]. So far, the input data was restricted to the information acquired from the robot joint angles and force information derived at the tip of the instruments for both the learning and the execution stage of automated tasks. A main issue arising with the reliance on this kind of data is the overall calibration of the kinematic chain, which is disturbed by many influences (like positional inaccuracy, distortion of the instruments and play). The instantiated concept of learningby-demonstration postulates the same capabilities of both teacher and trainee. Therefore, the awareness of the knowledge which can be perceived by the trainee is important. As the visual perception is used by humans as one of the primary modalities for spatial navigation and orientation, it is desirable to endow the trainee (more precisely: the technical system) with this capability. As a first step, we tackle the problem of positioning surgical instruments with high precision in the workspace what is a major contribution to the success rate of automated tasks. The proposed method is based on a two-step switching visual servoing scheme. In doing so, we overcome the above mentioned issues which may lead to imprecise executions of automated tasks or a miscarried skill transfer during the learning phase.

Visual servoing is a popular method to guide a robotic appendage using visual feedback from a camera system. A common scenario for visual servoing in medical applications is the automated guidance of an endoscope by an assistant robot [5], 
[6]. However, the required accuracy to align the camera is not very high and small variations do not affect the functionality or the security of the system. Furthermore, robotic endoscope holders represent eye-in-hand scenarios, involving just a single robot which is to be controlled and do not suffer as much from intrinsic calibration issues as complex multi-arm systems do. Krupa et al. [7] have demonstrated a system that automatically aligns surgical instruments equipped with an additional laserpointer to project an orientation pattern on the surface of the organs. Hynes et al. [8] have built a dual-arm system equipped with surgical instruments and a (conventional) stationary stereo camera to evaluate the prospects of visual servoed knot-tying. More recently, Nageotte et al. [9] have proposed a method for robot-assisted path following, using a $2 \mathrm{D}$ visual servoing scheme and depth estimation with markers applied on the instrument tip. In [10] they employ their approach to steer a circular needle with $4 \mathrm{DoF}$ instruments on an pre-computed path for optimal tissue piercing.

\section{Materials AND Methods}

Many works on the visual guidance of laparoscopic instruments are drawn on a simplified environment. Either the system lacks an endoscopic camera with strong distortions or the evaluation was performed within unrealistic dimensions of the workspace or distances between camera and instrument due to a missing multi-arm setup. While conclusions derived from simulations are comparably smooth, many awkward and unexpected details first occur, once the procedures are carried out in a complex setup. Therefore, the findings of this research project have been assessed within a realistic scenario of robotic surgery, whereas the presented solution is not limited to a specific number of robot arms or cameras.

\section{A. System Setup}

Our system comprises four relatively small robotic arms which are mounted upside-down on an aluminum gantry (cf. fig. 1). The arms are either equipped with force-feedback instruments or an endoscopic camera. Two different endoscopes are available: an analog stereo endoscope and a digital (mono) endoscope manufactured by Fujinon ${ }^{\mathrm{TM}}$ with chip-onthe-tip technology. Usually the Fujinon ${ }^{\mathrm{TM}}$ endoscope is used as an additional imaging device. A magnetic coupling mechanism allows quick mounting and dismounting of the tools without loosing the calibration against the rest of the system. The position and orientation of each manipulator is controlled via two PHANToM ${ }^{\mathrm{TM}}$ Premium 1.5 haptic input devices that have a switch to open and close the micro-gripper at the distal end of the instrument shaft. The user steers the instrument by moving a stylus pen that simulates the hand posture and feel of conventional surgical instruments. A crucial feature of the employed in/output devices is their capability of providing force feedback to the user. Translational forces are fed back by small servo motors incorporated into the device, and are used to steer the stylus pen in a certain direction while torque is neglected. This creates the impression of occurring forces, as the user is holding the pen at a certain posture. In order to

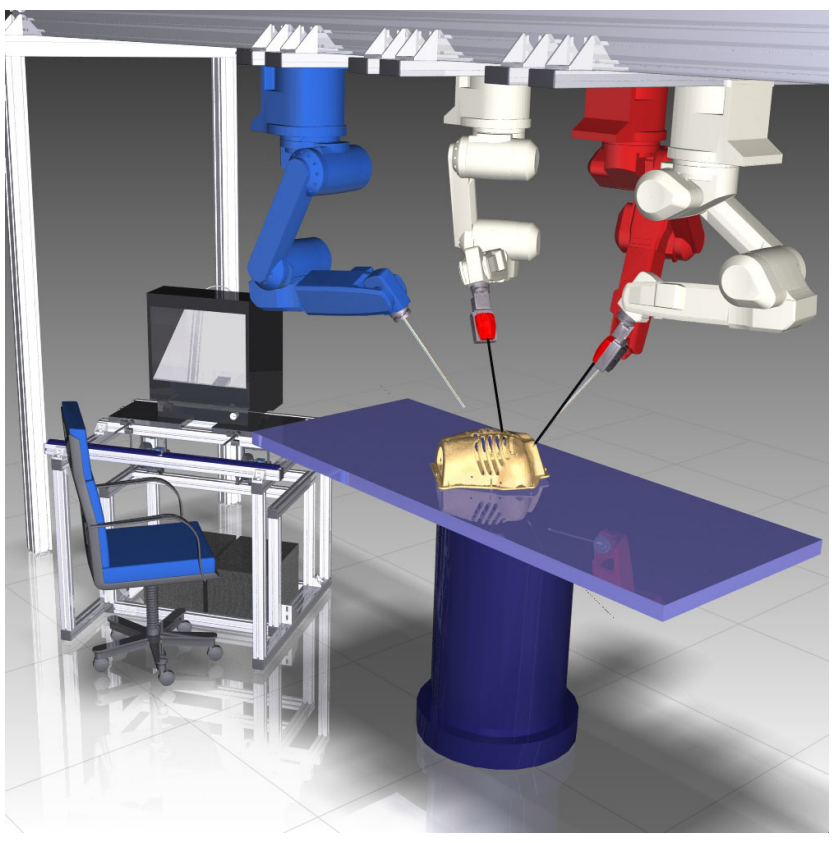

Fig. 1. Hardware Setup: Four ceiling mounted robots can be equipped with either a endoscope or different surgical instruments. The robot highlighted in red carries a stereo endoscope, the one highlighted in blue carries the Fujinon $^{\mathrm{TM}}$ endoscope.

be able to display realistic forces during operation, we have augmented the instruments with highly sensitive strain gauge sensors. The sensor readings are amplified and smoothed to stabilize the results. Apart from the manual interface, our system also comprises an interface for offline and real-time trajectory planning as well as collision detection.

\section{B. System Calibration ${ }^{1}$}

Unfortunately, many possible error sources contribute to a comparably high aberration between the real-world hardware and the underlying CAD models from the simulation environment. Camera calibration, exact mounting of the surgical instruments (concerning the magnet coupling) and even the instruments itself introduce quiet large errors. For instance, the flexibility and play of the carbon fiber shaft of the instruments and the gripper at the distal end may vary approximately $\pm 1.5 \mathrm{~cm}$ (cp. fig. 2(c)). Furthermore, the ceiling mounting of the robots afflicts several intrinsic aberrations, such as variations in the dimensions of the elements and errors of mounting angles. Since all errors sum up, the position of the end effector from a desired point in Cartesian coordinates deviates from the calculation of the emulation. In order to minimize the intrinsic error and to establish the transformations between the individual system components (such as the instrument, different robot bases, etc.) a precise calibration has to be

\footnotetext{
${ }^{1}$ To avoid unnecessary confusion we briefly declare the following conventions according to [11]: Let ${ }_{A}^{B} T$ be a homogeneous $4 \times 4$ matrix transforming a point $p_{1}$ from coordinate frame $A$ as $p_{1}^{A}$ into coordinate frame $B$ by $p_{1}^{B}={ }_{A}^{B} T p_{1}^{A}$. A velocity $\xi$ in point $P$, expressed in the reference frame $F$, is denoted as ${ }_{P}^{F} \xi$
} 


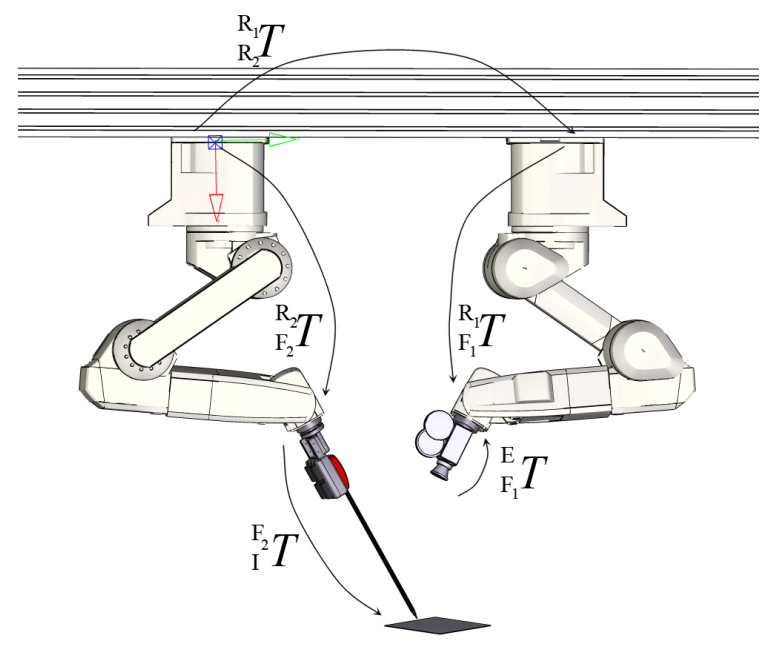

(a) System Calibration Overview

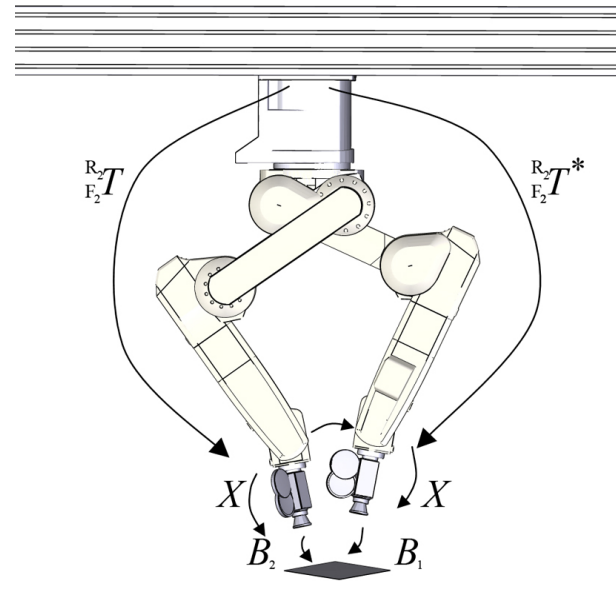

(b) Hand-Eye Calibration

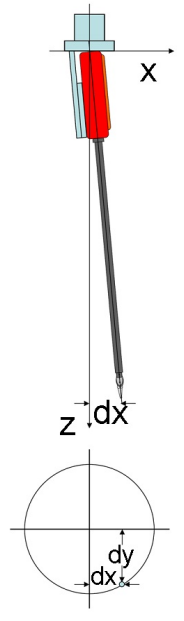

(c) Excentricity

Fig. 2. System Calibration. Figure 2(a) depicts a schematic overview of the system calibration, whereas the hand-eye calibration is exemplified in illustration 2(b). Figure 2(c) shows excentricity and play of the instrument shaft.

performed. An important issue for the acceptance of robotic systems in the operating theater are pre-calibrated components to avoid complicated or long-lasting procedures during an intervention or ahead. The calibration routine for the system components is exemplified next.

1) Robot Calibration: As mentioned above, the robots are mounted on a gantry, assembled of profiled girders. Particularly the coplanarity of the robot's base relative to its attachment cannot be guaranteed and is hardly to be measured. In order to overcome intrinsic variations of the single aluminum elements and errors of mounting angles a calibration between each of the robot basements is performed.

To align the basements of two robots $R_{1}$ and $R_{2}$ we employ the following error model:

$$
{ }_{R_{1}}^{0} T \cdot{ }_{C}^{R_{1}} T={ }_{R_{2}}^{0} T \cdot{ }_{C}^{R_{2}} T
$$

In this equation ${ }_{R_{1}}^{0} T$ is the position of the base of the robot $R_{1}$, expressed in global coordinates. In order to measure the relative displacement between the robots a calibration frame $C$ in global coordinates is defined and the position and orientation of this frame is measured in local coordinates of each robot. The frame can be replicated by mounting a precisely manufactured calibration trihedron of known size on the flange of both robots. A number of varying points $M=\left(p_{1}, \ldots, p_{i}\right)$ are labeled on a checkerboard calibration plate, which is positioned in-between the robots and can be reached by all arms (figure 2(a)). The tool tip of the calibration trihedrons of the robots $R_{1}$ and $R_{2}$ is then driven to all points and the corresponding relative transform (e.g. ${ }_{C}^{R_{2}} T$ ) between the basis can be determined by touching those points. The rotational part can then be written as follows:

$$
{ }_{R_{1}}^{R_{2}} R={ }_{C}^{R_{2}} T \cdot{ }_{C}^{R_{1}} T^{-1},
$$

whereas the translation is calculated between all points, considered in both coordinate frames:

$$
{ }_{R_{1}}^{R_{2}} t=\frac{1}{|M|} \sum_{M}\left(p_{i}^{R_{2}}-{ }_{R_{1}}^{R_{2}} R \cdot p_{i}^{R_{1}}\right)
$$

Mounting displacements of the robots are not the only source of errors in the system. If we go further down, we find that also the attachment of the instruments bears certain variances. Fortunately, hand-eye calibration is a well-known problem in robotics [12]. One way to calculate the displacement of an attached endoscope with respect to the flange of the robot, is to solve ${ }_{F_{2}}^{R_{2}} T \cdot X=X \cdot{ }_{F_{2}}^{R_{2}} T^{*}$ (compare fig. 2(b)).

2) Surgical Instrument Calibration: Another error source is the magnetic clutch and the instruments themselves. The possible play of the end effector is due to the flexibility of the used carbon fiber material. In order to determine and compensate the errors a handy-eye calibration could be employed, but the method would introduce two issues in the context of medical procedures: on one hand it is difficult to create a calibration pattern which can be precisely reached by the forceps or attached to the shaft. On the other hand, it is not possible to perform the calibration in the sterile environment of an operating room. The proposed method allows a precalibration of every instrument, which can be applied to the system previous to the intervention. To compensate the excentricity, an approximation which simplifies the calculation and applies only to small angles is used. An aberration $d x$ and $d y$ from the center will lead to a positional error or approximately $\sqrt{d x^{2}+d y^{2}}$. The parameters shown in fig. 2(c) can be found by positioning the instrument over a planar surface with the $z$-axis of the robot's tool system normal to the surface. By rotating the end effector about $360^{\circ}$ a circular path is described and the relevant parameters can be determined. In order to compensate for this excentricity, the 
found correctional transformation has to be applied to the end effector prior to the calculation of the inverse kinematics of the robot.

3) Endoscopic Camera Calibration: State of the art endoscopes offer physicians a wide-angled field of view which is imperative for minimally invasive interventions. In order to determine the projective parameters of the camera system a calibration procedure is performed a priori. Unfortunately, endoscopic cameras often suffer from inherent barrel distortions (see fig. 5(a)) and are delicate to calibrate. According to [13], algorithms for endoscope calibration can barely improve the accuracy.

\section{Visual Servo Control}

So far, we determined all transformations between the system components. Visual servoing is a popular method to guide a robotic appendage using visual feedback from a camera system. A good introduction is given by Chaumette and Hutchinson [14], [15]. In general, visual servoing can roughly be divided into two categories: position-based visual servoing control (PBVS), in which a Cartesian coordinate is estimated from image measurements and image-based visual servoing (IBVS) approaches, which seek to extract features directly from the images. In general, the accuracy of imagebased methods for static positioning tasks is less sensitive to calibration than PBVS [16], [17]. However, a practical difficulty during the alignment of surgical instruments with a desired position lies in the fact that the instrument is not necessarily in the field of view of the camera and no image-features can be extracted. In order to command surgical instruments with a high precision to a desired position, we propose a switching servoing scheme. First, the instrument has to be driven to the target Cartesian coordinate which is in sight of the camera, employing position-based servoing. Subsequently, we switch to image-based servoing to overcome the remaining error. The calibration method introduced above allows for a reconstruction of a $3 \mathrm{D}$ point in space and for the generation of movement commands for the robot which holds the instrument. Since a calibration will never be perfect and numerous parameters afflict the overall accuracy, the pose reached with PBVS is not totally congruent with the desired position. Image-based servoing does not depend as much on calibration as the error is reduced directly in image pixels. The proposed switching scheme is not only necessary to drive the instruments into the field of view of the camera, but also has a positive effect on the convergence characteristic of the image-based part.

Given a target pose or position that the robot is to reach, visual servoing aims to minimize an error $e(t)$, typically defined by

$$
e(t)=s(m(t), a)-s^{*}
$$

where $s^{*}$ represents the target pose, $s(m(t), a)$ the measured pose, $m(t)$ the measured image feature points and $a$ any additional knowledge needed, such as information from the camera calibration. The function $s(m(t), a)$ characterizes the end point of the tool tip of an instrument carried by the robot.
In position-based visual servoing the position of the tracked features is extracted from the camera image coordinates and projected to the world frame by the mapping $a$ determined during camera calibration. The target position can be extracted from image features in a similar way. While PBVS minimizes the error $e(t)$ in the world coordinate system and the camera is treated as a $3 \mathrm{D}$ positioning sensor, IBVS directly tries to find a mapping from the error function to a commanded robot motion.

1) Instrument Control: As mentioned above, PBVS is used to drive the instrument to a reconstructed point which is located within the view of the camera. As soon as this point is reached, the remaining distance to the target goal is minimized in image coordinates. In many IBVS scenarios the camera is directly attached to the robot which is to be commanded (eye-in-hand configuration) and therewith the velocity of the camera $\xi$ is calculated. In our setup, the instrument and the endoscope is carried by two different robots and the calculated velocity $\xi$ has to be transformed to the robot which carries the instrument. We first refer to the common case and the mapping of velocities is exemplified later on.

Only a single image feature, for instance the tip of an instrument or a needlepoint, is tracked in both left and right camera coordinates. The feature vector $s=\left(x_{L}, x_{R}\right)^{T}=$ $\left(u_{L}, v_{L}, u_{R}, v_{R}\right)$ comprises these coordinates:

$$
s(t)=\left[\begin{array}{l}
u(t) \\
v(t)
\end{array}\right]
$$

Its derivative $\dot{s}(t)$ is referred to as image feature velocity. It is linearly related to the camera velocity $\xi=\left[\begin{array}{ll}v & \omega\end{array}\right]^{T}$, which is composed of linear velocity $v$ and angular velocity $\omega$. The relationship between the time variation of the feature vector $s$ and the velocity in Cartesian coordinates $\xi$ is then established by

$$
\dot{s}=L_{s} \xi
$$

where $L$ is the interaction matrix or image Jacobian [14]. The interaction matrix $L_{x}$ related to an image point $x=(u, v)$ reads as follows:

$$
L_{x}=\left[\begin{array}{cccccc}
-\frac{1}{z} & 0 & \frac{u}{z} & u v & -\left(1+u^{2}\right) & v \\
0 & -\frac{1}{z} & \frac{v}{z} & 1+v^{2} & -u v & -u
\end{array}\right]
$$

In the matrix $L$, variable $z$ represents the depth of a point relative to the camera frame. There exist different ways to approximate the value of $z$, for example via triangulation in a stereo setup or via pose estimation. Most of the existing methods assume an calibrated camera, even if the impact of the calibration is not very high. Few systems even assume a constant depth of the tracked feature and therewith a constant image Jacobian. Though a stereo camera system is on hand, it is not used for the depth estimation. In fact, variable $z$ is estimated via the kinematic chain of the system, which allows for a calculation of the instrument in space. Using this method, $L$ can be updated on-line and the approach can easily be transfered to miscellaneous camera system. Hence, the Fujinon ${ }^{\mathrm{TM}}$ endoscope can be integrated as an additional image sensor, providing a different view on an object. Using 


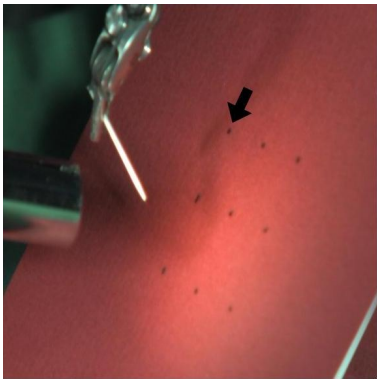

(a) initial position

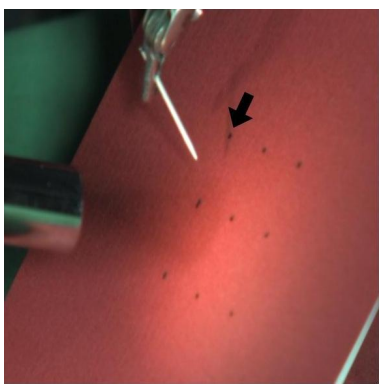

(c)

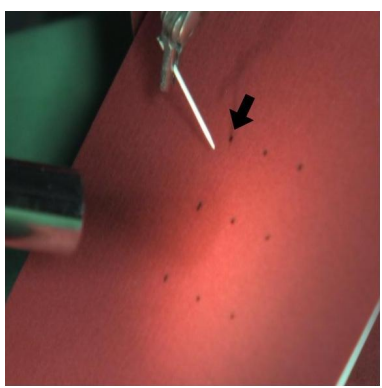

(e)

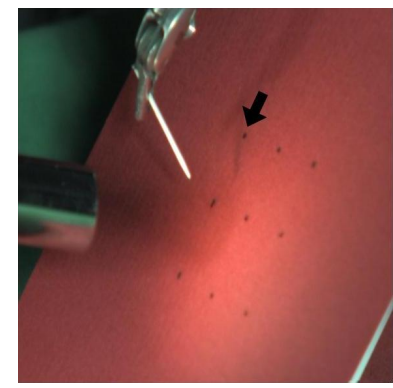

(b)

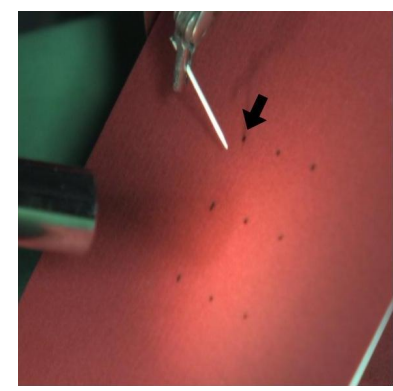

(d)

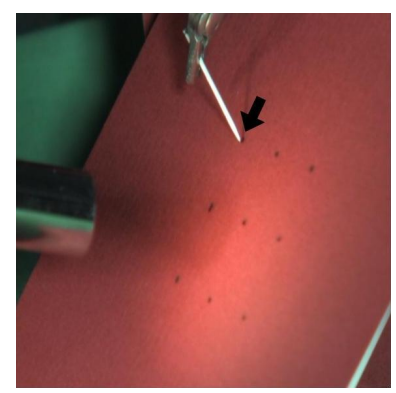

(f) final position
Fig. 3. Sequence of images during image-based visual servoing. The target position is set to the upper left point, out of the nine marked features. Figure 3(a) shows the initial state while the needle reached its final position in figure 3(f). Figures 3(b) - 3(e) illustrate the progression. The images are taken by an additional camcorder.

equations (4) and (6) we immediately obtain $\dot{e}=L_{e} \xi$ and our final control law

$$
\xi=\lambda L_{e}^{+} e
$$

where $\lambda$ is a positive gain factor and $L_{e}^{+}$the Moore-Penrose pseudo-inverse of $L_{e}$.

As mentioned above, a single visual feature $s$ is tracked in the left and right images, referred to as $\dot{x}_{L}=\left(\dot{u}_{L}, \dot{v}_{L}\right)$ and $\dot{x}_{R}=\left(\dot{u}_{R}, \dot{v}_{R}\right)$ and equation (6) is rewritten as

$$
\left[\begin{array}{l}
\dot{x}_{L} \\
\dot{x}_{R}
\end{array}\right]=\left[\begin{array}{l}
L_{L} \\
L_{R}^{R} V
\end{array}\right] \xi_{L}
$$

The spatial motion transform $V$ to transform velocities expressed in the right camera frame $R$ to the left camera frame $L$ is given by

$$
{ }_{L}^{R} V=\left[\begin{array}{cc}
{ }_{L}^{R} R & S(t){ }_{L}^{R} R \\
0 & { }_{L}^{R} R
\end{array}\right]
$$

where $S(t)$ is the skew symmetric matrix associated to the linear transformation vector $t$ and where $(R, t)$ is the transform from the left to the right camera frame.

2) Trocar Kinematics: In MIS it is indispensable to keep the instruments inside the boundaries of the insertion points in the body of the patient to ensure that no tissue is harmed. The Cartesian coordinate of the fulcrum is well-known in our setting. Constraining $6 \mathrm{DoF}$ of the robot to $4 \mathrm{DoF}$ at the trocar $T$, all motions perpendicular to the instrument shaft in $T$ are interdicted and two degrees of freedom are determined by the port. The velocities at the trocar point ${ }_{T}^{T} \xi=\left({ }_{T}^{T} v,{ }_{T}^{T} \omega\right)^{T}$ and the velocities of the instruments tip ${ }_{I}^{I} \xi=\left({ }_{I}^{I} v,{ }_{I}^{I} \omega\right)^{T}$ are related as follows:

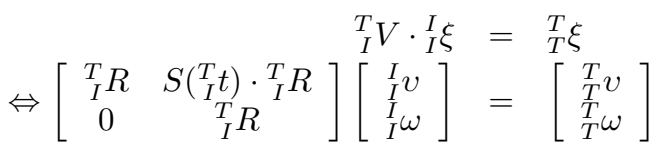

Assuming a straight shaft, ${ }_{I}^{T} R$ is the identity matrix and $t=$ $(0,0, d)^{T}$ with $d$ being the insertion depth of the instrument. Since only the $z$-direction (the direction of the shaft, 2(c)) is free to move, the linear velocity at the insertion point is donated by $v_{I}^{I}=\left(0,0, v_{I, z}^{I}\right)$. Solving (11) yields to

$$
{ }_{I}^{I} \omega_{x}=-\frac{{ }_{I}^{I} v_{y}}{d} \quad \text { and } \quad{ }_{I}^{I} \omega_{y}=\frac{{ }_{I}^{I} v_{x}}{d}
$$

\section{RESUlTS}

With the help of the introduced experimental platform we performed several tasks to measure the achieved precision. At this juncture, the position-based method and the image-based servoing were considered separately to clarify the improvement of the switching scheme. The instrument was holding a needle in its jaws which should align with the target. The tracking of the pinpoint was performed by a frame-by-frame color segmentation in HSV space. The PBVS was evaluated on about 40 points, labeled on a checkerboard and positioned freely in space. To investigate the influence of strong distortions the Fujinon ${ }^{\mathrm{TM}}$ endoscope features a good platform (cp. 5(a)). Since it is a single-chip camera, stereoscopy has to be simulated by displacing the camera with the robot arm. Admittedly this method may introduce some additional errors, but it is our only possibility to research on strong deformations. We also repeated the experiment with a stereo endoscope.

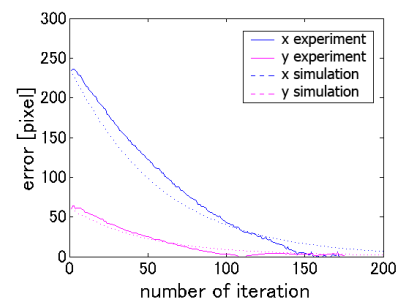

(a)

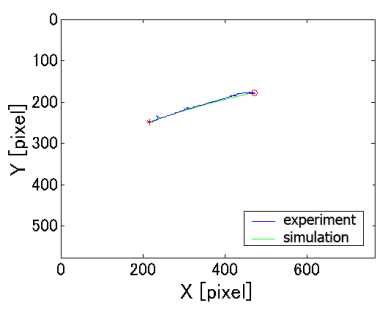

(b)
Fig. 4. Performance of IBVS. 4(a) Convergence of the error, 4(b) real-world trajectory vs. simulation. 
However, the better performance can basically be ascribed to the more simple camera geometry. The aberration between the desired and the reached position was measured in $x, y$ and $z$ direction and is visualized in fig. 5(b). Over a broad range, the positioning error ranges from $3-7 \mathrm{~mm}$. As expected, the precision degrades as the reconstructed points are close to the image margins. The rising error at the front and rear edges of the plot result from the working area of a reliable $3 \mathrm{D}$ reconstruction with the endoscope (approx. $1.5 \mathrm{~cm}-6 \mathrm{~cm}$ ). The image-based part was verified in a similar way, with the instruments being visible for the camera at the start time. Nine different targets were identified in image coordinates. The tip of the needle was driven subsequently to all nine desired positions, whereas the positioning error was evenly distributed over the entire image and did not exceed $1.3 \mathrm{~mm}$. The convergence behavior of the image-based servoing was simulated in advance. Figure 4 depicts the simulation results as well as data recorded during the experiments. Single snapshots taken during the positioning task are shown in figure 3 .

\section{CONCLUSION}

We have combined position-based and image-based visual servoing into a switching scheme in order to precisely maneuver surgical instruments in a complex setup. Both methods are essential to command the robot in the case that the surgical instrument is not in the field of view of the camera. An overall system calibration was performed to establish all necessary transformations and to minimize intrinsic inaccuracies. The approach was verified in a realistic multi-arm setup for heart surgery. As the position-based method just allows for a coarse positioning within $3-7 \mathrm{~mm}$, the image-based part is able to further minimize the error under $1.3 \mathrm{~mm}$. Considering the presented approach, image processing and tracking is under ongoing work to improve robustness against noise and the loss of image features. The stability of the tracking is crucial for a fast convergence in IBVS and turned out to be the main weakness in our setting. The stereo tracking is also affected by the small baseline of the endoscope. Deflections in the plot of fig. 4 are often caused by tracking imprecisions and noise. The combination of several cameras (such as the

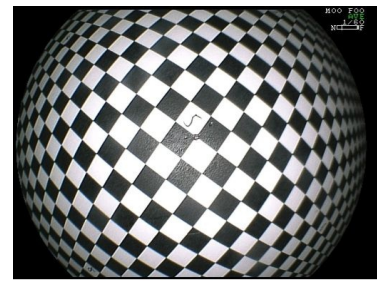

(a) Fujinon ${ }^{\mathrm{TM}}$ camera distortion

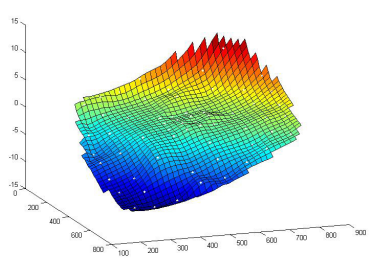

(b) error plot of PBVS
Fig. 5. Position-Based VS: Fig 5(a) clarifies the strong distortions of the endoscopic camera by capturing a checkerboard. Fig. 5(b) shows the Cartesian positioning error. The error increases when the point is located close to the borders of the range where $3 \mathrm{D}$ information can be extracted reliably (front and rear edges). stereo endoscope and the Fujinon ${ }^{\mathrm{TM}}$ together) could stabilize tracking results. In order to integrate the orientation of the gripper at the distal end of the instrument, partitioned IBVS approaches could improve the system behavior. So far, an obstacle-free environment was assumed. For the application in more complex automated tasks, such as tissue piercing, a detection and modeling of the environment has to be included.

\section{REFERENCES}

[1] A. Garcia-Ruiz, M. Gagner, J. H. Miller, C. P. Steiner, and J. F. Hahn, "Manual vs robotically assisted laparoscopic surgery in the performance of basic manipulation and suturing tasks," Arch Surg, vol. 133, no. 9, pp. 957-961, 1998.

[2] H. Mayer, I. Nagy, A. Knoll, E. Braun, R. Bauernschmitt, and R. Lange, "Haptic feedback in a telepresence system for endoscopic heart surgery," MIT PRESENCE: Teleoperators and Virtual Environments, vol. 16, no. 5, pp. 459-470, 2007.

[3] H. Mayer, I. Nagy, A. Knoll, E. Braun, R. Lange, and R. Bauernschmitt, "Adaptive control for human-robot skilltransfer: Trajectory planning based on fluid dynamics," in Proceedings of the IEEE International Conference on Robotics and Automation, Rome, Italy, April 2007, pp. 1800-1807.

[4] H. Mayer, I. Nagy, A. Knoll, E. Braun, and R. Bauernschmitt, "Humancomputer interfaces of a system for robotic heart surgery," in Proceedings of the Second IASTED International Conference on HumanComputer Interaction, Chamonix, France, March 2007, pp. 31-36.

[5] A. Casals, J. Amat, and E. Laporte, "Automatic guidance of an assistant robot in laparoscopic surgery," Robotics and Automation, 1996. Proceedings., 1996 IEEE International Conference on, vol. 1, pp. 895-900 vol.1, Apr 1996.

[6] G.-Q. Wei, K. Arbter, and G. Hirzinger, "Real-time visual servoing for laparoscopic surgery. controlling robot motion with color image segmentation," Engineering in Medicine and Biology Magazine, IEEE, vol. 16, no. 1, pp. 40-45, Jan.-Feb. 1997.

[7] A. Krupa, J. Gangloff, C. Doignon, M. de Mathelin, G. Morel, J. Leroy, L. Soler, and J. Marescaux, "Autonomous 3-d positioning of surgical instruments in robotized laparoscopic surgery using visual servoing," Robotics and Automation, IEEE Transactions on, vol. 19, no. 5, pp. 842-853, Oct. 2003.

[8] P. Hynes, G. Dodds, and A. Wilkinson, "Uncalibrated visual-servoing of a dual-arm robot for mis suturing," Biomedical Robotics and Biomechatronics, 2006. BioRob 2006. The First IEEE/RAS-EMBS International Conference on, pp. 420-425, Feb. 2006.

[9] F. Nageotte, P. Zanne, C. Doignon, and M. de Mathelin, "Visual servoing-based endoscopic path following for robot-assisted laparoscopic surgery," in Intelligent Robots and Systems, 2006 IEEE/RSJ International Conference on, Oct. 2006, pp. 2364-2369.

[10] — " "Stitching planning in laparoscopic surgery: Towards robotassisted suturing," The International Journal of Robotics Research, p. 0278364909101786, 2009.

[11] M. W. Spong, S. Hutchinson, and M. Vidyasagar, Robot Modeling and Control. Wiley \& Sons, Inc., 2005.

[12] Y. Shiu and S. Ahmad, "Calibration of wrist-mounted robotic sensors by solving homogeneous transform equations of the form $\mathrm{ax}=\mathrm{xb}$," Robotics and Automation, IEEE Transactions on, vol. 5, no. 1, pp. 16-29, Feb 1989.

[13] C. Wengert, M. Reeff, P. Cattin, , and G. Szekely, "Fully automatic endoscope calibration for intraoperative use," in Bildverarbeitung für die Medizin, 2006.

[14] F. Chaumette and S. Hutchinson, "Visual servo control. basic approaches." Robotics \& Automation Magazine, IEEE, vol. 13, no. 4, pp. 82-90, Dec. 2006.

[15] - "Visual servo control. advanced approaches." Robotics \& Automation Magazine, IEEE, vol. 14, no. 1, pp. 109-118, March 2007.

[16] B. Espiau, "Effect of camera calibration errors on visual servoing in robotics," in The 3rd International Symposium on Experimental Robotics. London, UK: Springer-Verlag, 1994, pp. 182-192.

[17] S. Hutchinson, G. Hager, and P. Corke, "A tutorial on visual servo control," IEEE Transactions on Robotics and Automation, vol. 12, pp. 651-670, 1996. 